\title{
1 Interpretable machine learning models for 2 single-cell ChIP-seq imputation
}

3 Steffen Albrecht ${ }^{1,2}$, Tommaso Andreani 1,3,4, Miguel A. Andrade-Navarro ${ }^{1}$, Jean-Fred 4 Fontaine ${ }^{1, *}$

1 Institute of Organismic and Molecular Evolution (iOME), Johannes Gutenberg University Mainz, Mainz D-55128, Germany

2 Current Address: Institute of Physiology, University Medical Center of the Johannes Gutenberg University Mainz, Mainz D-55128, Germany

3 Institute of Molecular Biology, Mainz D-55128, Germany

4 Current Address: AI and Deep Analytics, Sanofi-Aventis, Frankfurt D-65929, Germany

* to whom correspondence should be addressed: fontaine@uni-mainz.de

\section{Abstract}

Motivation: Single-cell ChIP-seq (scChIP-seq) analysis is challenging due to data sparsity.

High degree of data sparsity in biological high-throughput single-cell data is generally handled lacking. We present SIMPA, a scChIP-seq data imputation method leveraging predictive information within bulk data from ENCODE to impute missing protein-DNA interacting regions of target histone marks or transcription factors.

Results: Imputations using machine learning models trained for each single cell, each target, and each genomic region accurately preserve cell type clustering and improve pathway-related gene identification on real data. Results on simulated data show that 100 input genomic regions are already enough to train single-cell specific models for the imputation of thousands of undetected regions. Furthermore, SIMPA enables the interpretation of machine learning 
models by revealing interaction sites of a given single cell that are most important for the imputation model trained for a specific genomic region. The corresponding feature importance values derived from promoter-interaction profiles of $\mathrm{H} 3 \mathrm{~K} 4 \mathrm{me} 3$, an activating histone mark, highly correlate with co-expression of genes that are present within the cell-type specific pathways. An imputation method that allows the interpretation of the underlying models facilitates users to gain an even deeper understanding of individual cells and, consequently, of sparse scChIP-seq datasets.

Availability and implementation: Our interpretable imputation algorithm was implemented in Python and is available at https://github.com/salbrec/SIMPA

\section{Introduction}

The discovery of protein-DNA interactions of histone marks and transcription factors is of great importance in biomedical studies because of their impact on the regulation of core cellular processes such as chromatin structure organization and gene expression. These interactions are measured by chromatin immunoprecipitation followed by high-throughput sequencing (ChIPseq). Public data from the ENCODE portal, which provides a large collection of experimental bulk ChIP-seq data, has been used for comprehensive investigations providing insights into epigenomic processes that affect chromatin 3D-structure, chromatin state, and gene expression, to name just a few (Consortium and others, 2012).

Recently developed protocols for scChIP-seq are powerful techniques that will enable indepth characterization of those processes at single-cell resolution. ChIP-seq was successfully

44 performed on single cells with sequencing depth as low as 1,000 unique reads per cell, reflecting the low amount of cellular material that can be obtained from only one single cell 
46 (Rotem et al., 2015). Even though this low coverage leads to sparse datasets, scChIP-seq data

47 has enabled the study of biological systems that cannot be investigated with bulk ChIP-seq

48 applied for millions of cells, for example, the differences between drug-sensitive and drug-

49 resistant breast cancer cells (Grosselin et al., 2019).

50 Nevertheless, the analysis of single-cell assays is strongly affected by the sparsity of data.

51 In the context of ChIP-seq, sparsity means no signal observed for numerous genomic regions

52 without the possibility to explain whether this is real or due to low sequencing coverage.

53 Notably, sparsity may disable the investigation of functional genomic elements that could be

54 of crucial interest. Hence, an imputation method is needed to complete sparse scChIP-seq

55 datasets while preserving the identity of each individual cell.

The first published imputation method for NGS epigenomic signals was ChromImpute

57 (Ernst and Kellis, 2015), later followed by (Durham et al., 2018), an improved method for the imputation of signal tracks for several molecular assays in a biosample-specific manner (biosample refers to the specific tissue or cell-type). The challenge of transcription factor binding site prediction was approached, for example, using deep learning algorithms on sequence position weight matrices (Qin and Feng, 2017), and more recently by the embedding of transcription factor labels and k-mers (Yuan et al., 2019). With the aim to complete the

63 ENCODE portal with imputed bulk experiments, Schreiber et al. implemented the method

64 Avocado, which extends the basic concept of PREDICTD by deep neural networks (Schreiber, Durham, et al., 2020). Avocado was also validated on ChIP-seq data from both histone marks and transcription factors (Schreiber, Bilmes and Noble, 2020). Such methods show the

67 successful application of machine learning algorithms and mathematical approaches in predicting epigenomic signals such as transcription factor binding activity. However, their 
scope, being limited to either imputation of missing bulk experiments or sequence-specific binding site prediction, hampers their application to single-cell data.

The challenge of imputation for sparse datasets from single-cell assays has been extensively approached for single-cell RNA-seq (scRNA-seq) used to quantify gene expression at singlecell resolution (Ronen and Akalin, 2018; Zhang and Zhang, 2018; Peng et al., 2019; Chen et al., 2020; Elyanow et al., 2020; Jeong and Liu, 2020; Tang et al., 2020; Ye et al., 2020; Zhu and Anastassiou, 2020). In this context, similarly to scChIP-seq data, sparsity is described by dropout events, which are transcripts having a transcription rate of zero without knowing if the corresponding gene is not expressed at all or if the expression rate is not detected due to technical limitations (Tang et al., 2020). The question arises if these methods can be easily adapted for imputation of scChIP-seq data. However, there are crucial differences between the application of RNA-seq and ChIP-seq techniques that must be considered regarding the development of a method for scChIP-seq imputation.

First, in RNA-seq the set of relevant genomic regions, defined by the species-specific transcripts, is more limited. For a ChIP-seq profile, the regions of potential interest may originate from any position in the genome and cannot be defined in advance. To simplify the analysis, in scChIP-seq imputation the genome can be organized in non-overlapping genomic windows (bins) of a certain size. At $5 \mathrm{~kb}$ resolution, this binning concept results in more than 600,000 possible regions in the human genome, a number that is much higher than the number of transcripts considered in the RNA-seq context.

The second main difference is that scChIP-seq interactions are usually represented by a Boolean value describing the presence or absence of a significant enrichment of sequencing reads defining a peak, while RNA-seq datasets contain transcription rates. Consequently, the application of scRNA-seq imputation methods on scChIP-seq data might be less appropriate. 
In contrast, imputation methods for chromatin accessibility profiles from single-cell ATAC-

94 seq (single-cell Assay for Transposase-Accessible Chromatin using sequencing, scATAC-seq) are potentially more transferable to scChIP-seq imputation as their data representation is more similar. A few methods exist that implement imputation for scATAC-seq, though none of them was tested on scChIP-seq data so far. Methods such as SCALE (Xiong et al., 2019), FITs (Sharma et al., 2020) and scOpen (Z. Li et al., 2019) have been shown to outperform scRNAseq methods to impute scATAC-seq data. These methods complement each other with respect to the different approaches they implement, however, they share the common concept of imputing the missing values within a sparse matrix defined by the single cells (rows) and genomic bins (columns), and only bins are considered that were detected by at least one single cell if no further filtering is applied. Consequently, such methods can offer imputation only on regions that were observed in the single-cell dataset and it is likely that many important regions along the whole genome will be missed.

To overcome this limitation, we developed SIMPA, an algorithm for Single-cell ChIP-seq iMPutAtion, that uses bulk ChIP-seq datasets of the ENCODE project ("The ENCODE (ENCyclopedia Of DNA Elements) Project," 2004; Sloan et al., 2016). It was already shown that an additional bulk RNA-seq dataset can be used to improve the imputation for a sparse scRNA-seq dataset (Peng et al., 2019). Within SIMPA, publicly available bulk ChIP-seq data is turned into a reference set used to define potential bins to be imputed and then leveraged by machine learning models to compute specific imputation probabilities. Moreover, these models are interpretable and can be used to gain more insights into a given single-cell dataset, allowing the investigation of individual cells on a more detailed level. The interpretability is implemented by InterSIMPA, an extension of SIMPA, which takes a single cell as input together with a genomic position of interest and trains one classification model for the position to derive a probability which can be seen as an imputation score. More importantly, 
118 InterSIMPA ranks the genomic regions from the sparse single cell profile by their relevance

119 for the model. The ranked regions are enriched by detailed information and an importance score

120 describing the strength of their relationship with the given genomic position of interest. These

121 relationships can be interpreted as dependencies between genomic regions that could be part

122 of the gene regulatory network (e.g., between enhancers and promoters).

The basic reference-based imputation concept of SIMPA was first validated on simulated data and then on a real scChIP-seq dataset of the H3K4me3 and H3K27me3 histone marks in

B-cells and T-cells (Grosselin et al., 2019). The latter dataset allowed us to investigate the algorithm's capability of retaining the cell-type clustering and furthermore to assess the biological relevance of the imputed regions based on a pathway enrichment analysis. These validations were performed in comparison to a reference-free method represented by the aforementioned scATAC-seq analysis method SCALE, which applies a combination of

130 Gaussian mixture models and variational autoencoder (Xiong et al., 2019). Results from signaling pathways, and compared to gene co-expression data derived from the STRING database (Szklarczyk et al., 2019).

Unlike many other single-cell imputation methods, SIMPA leverages predictive information within bulk ChIP-seq data by combining the sparse input of one single cell and a collection of 


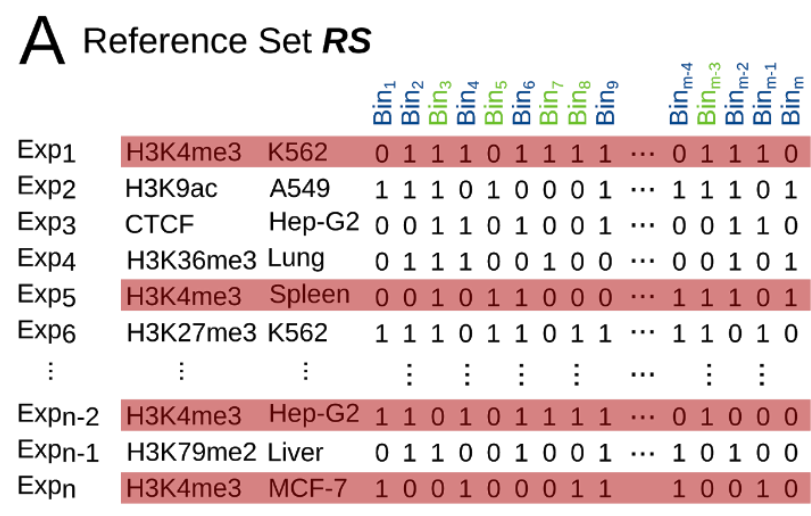

Bins observed in the sparse single-cell, SC (user provided)

Target: the histone mark or transcription factor, targeted by antibody in ScChIP (defined by user, e.g. H3K4me3)

Candidate bins $\boldsymbol{c}$, being potentially imputed

C Cross-Validations within H3K4me3 Data Areas under ROC-Curves (AUROC)

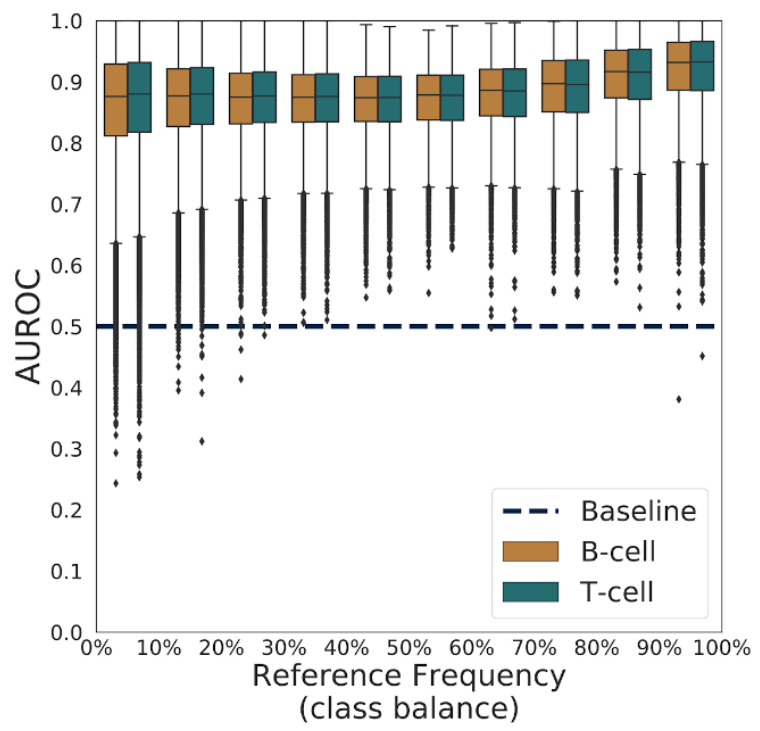

B Training Features TF and Candidate Bins $c$

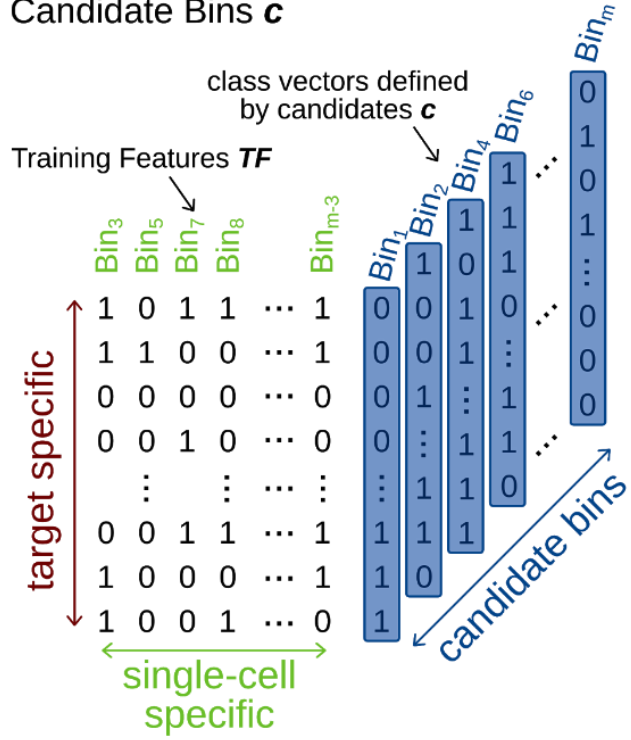

Areas under Precision-Recall Curves (AUPRC)

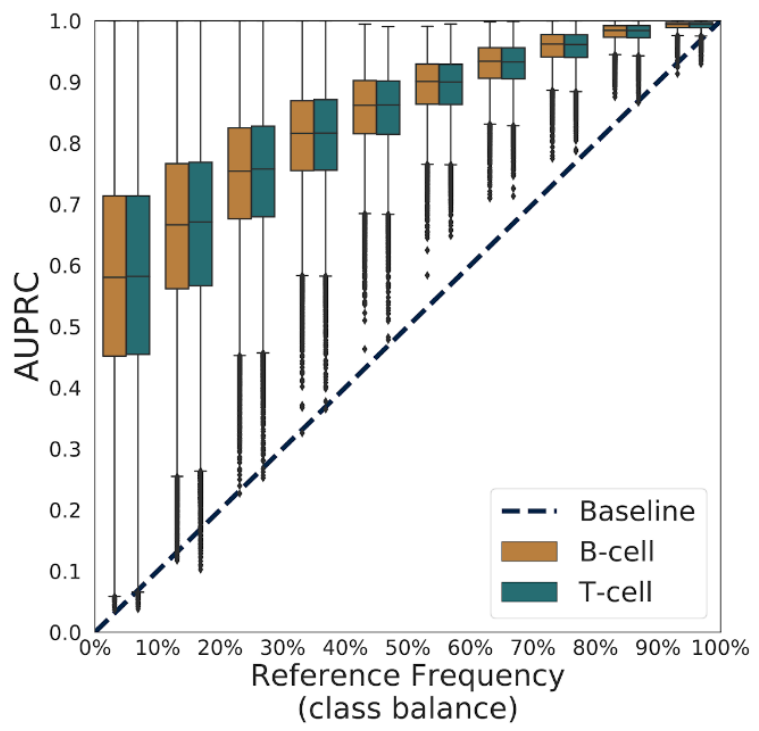

144 A. Identified ChIP-seq regions from bulk experiments were downloaded from ENCODE and 145 mapped to bins defined as non-overlapping and contiguous genomic regions of a defined length 146 (5 kb for H3K4me3 and $50 \mathrm{~kb}$ for H3K27me3) and covering the whole genome (the table). A 147 bin is given a value of 1 for a particular experiment if there is at least one ChIP-seq region in 
this experiment that overlaps the bin, 0 otherwise. In total 2251 ChIP-seq experiments for several targets (histone marks or transcription factors) performed in several biosamples (tissues and cell-lines) were downloaded from ENCODE portal and preprocessed. Depending on the target specified by the user, the target-specific reference set $\boldsymbol{R S}$ is then created and contains all experiments related to this target (red lines) and all bins observed for at least one of those experiments. $\boldsymbol{B}$. The single-cell specific training feature matrix $\mathbf{T F}$ is created as a subset of $\boldsymbol{R S}$ by selecting only bins observed within the given single cell (green columns). All other bins from $\boldsymbol{R S}$ are the candidate bins (c; blue columns) and define the class vectors consisting of the corresponding values in RS. For each candidate bin, a classification model is trained based on the training features and the class vector identifying associated experiments. C. Cross-validated evaluations of SIMPA's Random Forest performances to predict values of candidate bins in single cells within the H3K4me3 data. For each bin, a tenfold cross-validation was applied and summarized as Area under ROC-Curve (AUROC) or Area under Precision-Recall Curve (AUPRC) (y-axes). Results for all bins are represented by boxplots subdivided by class balance in the candidate bins (percentage of "1" values in the bin) (x-axis). The dashed lines describe the baseline performance expected from a random classification model: 0.5 for AUROC and equal to the class balance for AUPRC.

SIMPA produces results for each single cell of a scChIP-seq dataset by using machine learning models trained on a subset of the ENCODE data related to a selected target, that is the histone mark or transcription factor used in the single-cell experiment. Derived from this targetspecific subset, the classification features are defined by genomic regions detected in the single cell, while the class to predict is defined by a region observed in at least one target-specific bulk ENCODE experiment, but not in the single cell (Fig. 1B). In other words, by using this particular data selection strategy, SIMPA searches relevant statistical patterns linking (i) protein-DNA interacting regions across single-cell specific regions of the target-specific ENCODE data for different cell types to (ii) the presence or absence of a potential region for the given single cell. SIMPA's machine learning models are able to use those patterns to provide accurate predictions (Fig. 1C, Supplementary Note 3, and Fig. S1). Moreover, on the high-resolution H3K4me3 dataset, SIMPA achieved high recall rates for bins removed from 
177

178

179

180

181

182

183

184

185

186

187

188

189

190

191

192

193

194

195

196

197

198

single-cell profiles (Supplementary Note 4 and Fig. S2). The single-cell profiles used to create the training feature sets within the cross-validations were defined by the dataset from Grosselin et al. (Grosselin et al., 2019) called the real scCHIP-seq dataset below.

\section{Validation on simulated data}

In order to evaluate the algorithm's ability from few input bins (hundreds) to complete full data profiles (thousands of bins) of different protein targets and cell-types, we simulated sparse protein-DNA interaction profiles from the bulk ENCODE ChIP-seq experiments that are used as reference data by SIMPA. For the simulation, we took bulk experiments for different celltype-target combinations to define them as full single-cell profiles (origin) and down-sampled those profiles to simulate sparse single-cell profiles (from 100 to 1600 bins) (see

Supplementary Note 5 for details). Each simulated sparse profile was used as input for SIMPA and the output was compared to the origin. For the model training, the full origin profile was excluded from the reference training set in order to apply the default validation, called leaveout origin (LOO). Additionally, a more challenging validation strategy was applied in which all reference profiles for the same cell-type (biosample) were excluded, called leave-out celltype (LOCT).

For $\mathrm{H} 3 \mathrm{~K} 4 \mathrm{me} 3$, the most frequently investigated target in ENCODE, high area under ROCcurve values confirm that SIMPA is able to accurately recapitulate the original data from the simulated sparse profiles (Fig. 2A). Even if the cell-type-specific information is completely removed from the training set (LOCT), the performance is still high. Furthermore, these observations are confirmed when using precision-recall curves as performance measure (Fig. 2B), a highly relevant analysis given the imbalance in the validation sets (containing far fewer 


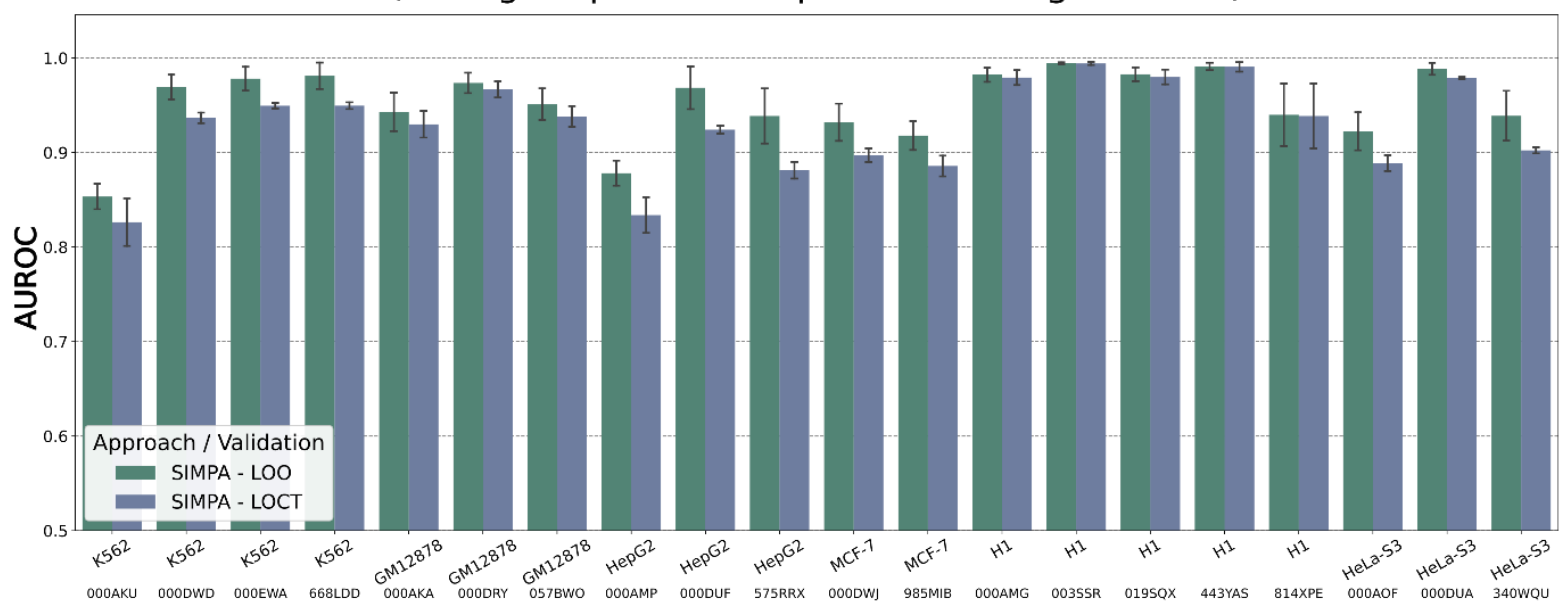

B

Origin

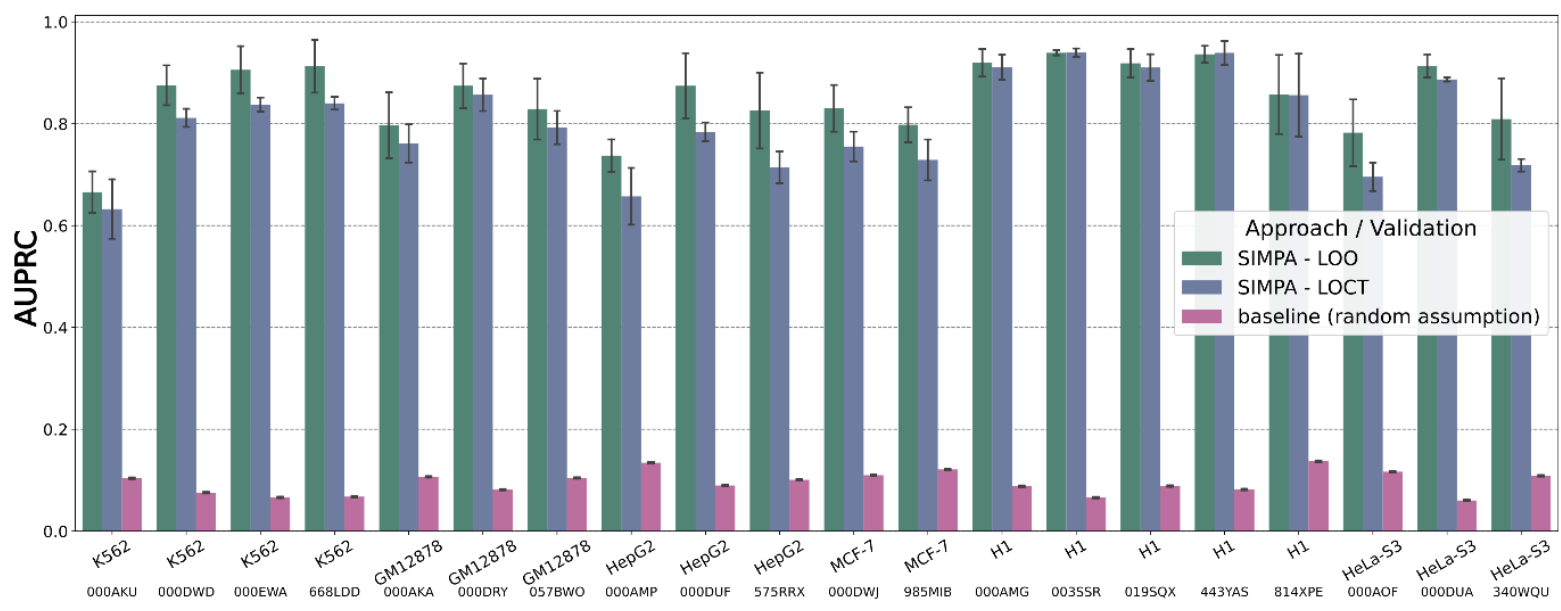

Origin

A. Using the origin profile for simulation as the validation set of true binding interactions, the area under ROC-curve (AUROC in y-axis) describes the capability of SIMPA to accurately impute and recapitulate the bulk experiment. The bars describe the mean AUROC and the error bars describe the standard deviation across multiple applications on sparse sets with different sizes. SIMPA was validated with two strategies, the default leave-out origin (LOO) and the extreme leave-out cell-type (LOCT). The $x$-axis labels indicate the cell-type of the origin profile and additionally the ENCODE accession to show which of the experimental dataset was used 
210 as origin. B. Same as in $\boldsymbol{A}$ but using the area under precision-recall curve (AUPRC in y-axis)

211 as performance measure. The pink bars show the class balance (fraction of positives in the

212 class feature) representing the random assumption as baseline to be expected from a primitive

213 classifier that randomly assigns the class values (according to (Saito Takaya AND

214 Rehmsmeier, 2015)).

215 In order to assess the single-cell specificity of SIMPA in this simulation, we compared each

216 fully imputed profile to its origin profile and also to a consensus profile representing

217 experimental datasets that are most similar to the origin (experimental profiles with same

218 protein target and same cell-type, see Supplementary Note 5 for details). Results show that

219 for most of the simulations ( $>95 \%$ ) the imputed profile is closer to the origin profile, hence

220 single-cell specific (Fig. 3A). Moreover, we observed that the origin profiles can be more

221 similar to the consensus profile (less specific) or less similar (more specific). When the origin

222 profiles are less specific, it is harder for SIMPA to achieve an imputed profile specific to the

223 origin (single-cell specific). However, for such cases in which the origin is quite close to the

224 consensus (Jaccard-Index > 0.65) the imputation is still single-cell specific, although with a

225 lower single-cell specificity value (Fig. 3B). 

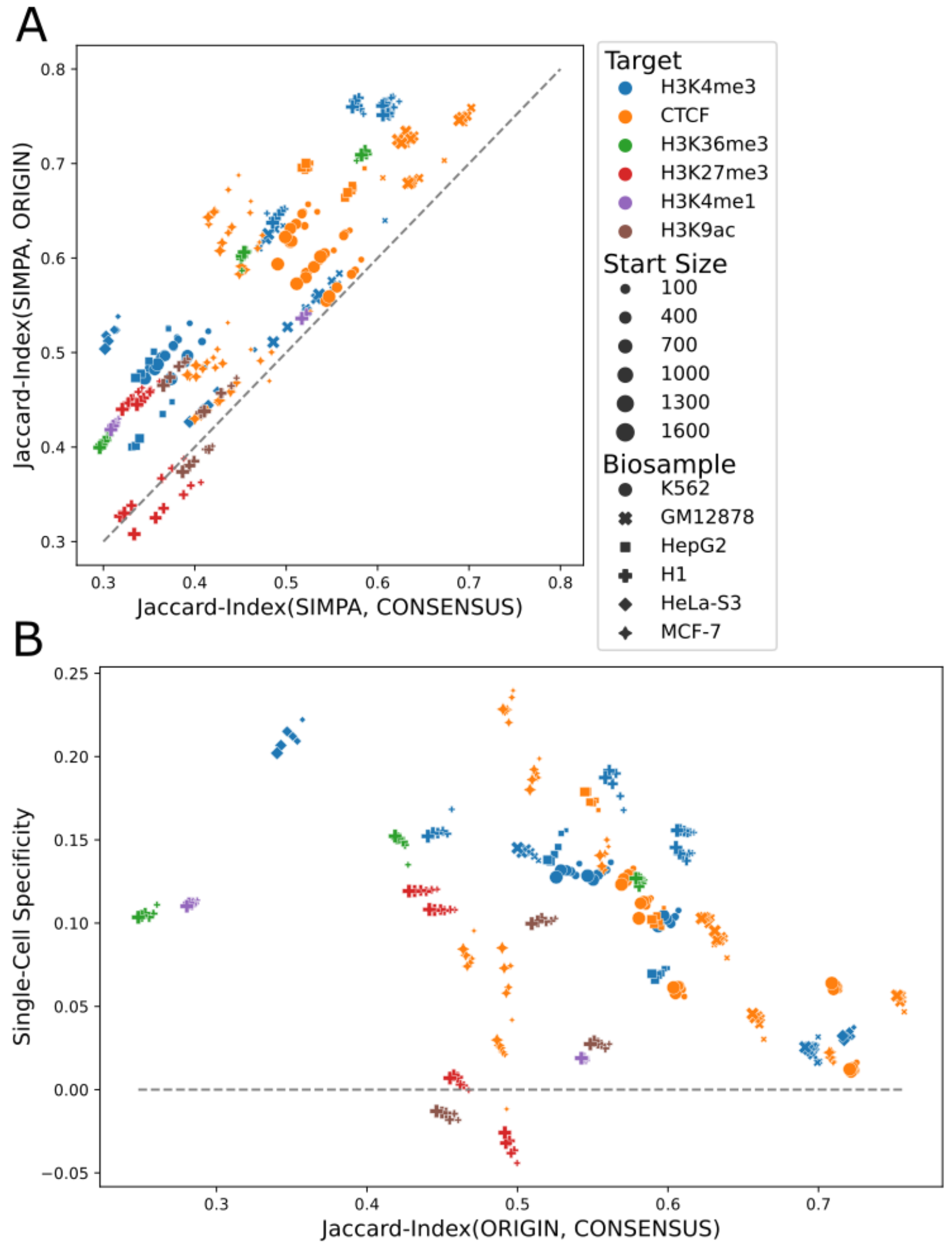

Figure 3 - Single-cell specificity analysis

228 A. The Jaccard-Index is used to compare the imputed profiles from SIMPA with the origin 229 profile used to create a simulated sparse profile and the consensus profile representing the 
230

231

232

233

234

235

236

237

238

239

240

241

242

243

244

245

246

247

248

249

250

251

252

253

remaining experiments available for the same biosample-target combination as the origin profile. The dashed line shows the balance line at which the imputed profile from SIMPA is neither closer to the origin nor to the consensus. Cases above the dashed line are those in which the imputed profile is single-cell specific, hence, closer to the origin than to the consensus. B. "Single-Cell Specificity" on the y-axis is defined as the difference between the imputed-to-origin similarity (y-axis in $\boldsymbol{A}$ ) and imputed-to-consensus similarity (x-axis in $\boldsymbol{A}$ ). Having the similarity between the origin and the consensus on the x-axis, this plot allows the visualization of the single-cell specificity in relation to how specific the origin is. The higher the similarity between the origin and consensus, the less specific is the origin profile and the harder the challenge to capture its specificity. Profiles, above the 0 line, are single-cell specific as they are closer to the origin than to the consensus.

Taken together, the simulation results show that models trained from a few bins accurately impute thousands of bins and show that completed profiles can be single-cell specific on real data even if the investigated cell-type is not represented by any of the bulk datasets in the reference set (leave-out cell-type validation).

\section{Model interpretability on real data}

Addressing one main aims of this study: to make models interpretable, we implemented an extension called InterSIMPA. Here we define interpretability as the possibility of obtaining information of potential biological relevance from the relationships observed between the training features (genomic regions) and an imputed region of interest. These relations can be expected to be part of the genomic regulatory network.

The training features are derived by InterSIMPA in the same way as for SIMPA but a single machine learning model is trained for a genomic position of interest defined by the user. Accordingly, one imputed probability is returned with information about the genomic regions 
from the single cell most important for the machine learning model. Finally, the algorithm reports the genes closest to these regions (Supplementary Note 6).

To demonstrate how interpretable imputation models can be used to expose more information from the sparse ChIP-seq profile of individual single cells, we use the single-cell 258 ChIP-seq dataset of $\mathrm{H} 3 \mathrm{~K} 4 \mathrm{me} 3$ interactions in B-cells and T-cells from Grosselin et al. 259 According to the given cell types, we focused on promoter regions of genes that are involved within the B-cell and T-cell receptor signaling pathways. The two gene sets contain 67 and 97 genes for the B-cell receptor and T-cell receptor signaling pathways, respectively, with an overlap of 44 genes. To focus on the genes that could be more specific to the cell-types under investigation, from the union of the two gene sets we selected 24 genes with frequency of their promoter regions lower than $20 \%$ in the corresponding H3K4me3-specific reference set, which means that their promoter has no detected interaction site for more than $80 \%$ of the ENCODE reference experiments for H3K4me3 in different cell-types and tissues (Supplementary Note 6).

As $\mathrm{H} 3 \mathrm{~K} 4 \mathrm{me} 3$ is an activating histone mark, we expected to observe interaction sites in the promoter regions of these genes. However, for many of those promoter regions, the H3K4me 3 binding is missing for most of the single cells in the sparse data (Fig. 4A). Our expectation that SIMPA is able to impute such regions in a cell-type-specific manner, is confirmed by comparing the imputed probabilities calculated by SIMPA for promoter regions of the 24 selected genes in single B- and T-cells (Fig. 4B). For most of the genes, the imputed probability 274 is higher when SIMPA is applied on single cells that are from the pathway-related cell-type. 275 Finally, we evaluated the interpretability of the 24 imputation models by comparing the feature 276 importance values of the extracted features from the single cell and co-expression values of the 277 feature-related genes with the gene of the imputed promoter (Fig. 4C). Co-expression data 
bioRxiv preprint doi: https://doi org/101101/2019.12 20.883983; this version posted July 12,2021 . The copyright holder for this preprin (which was not certified by peer review) is the author/funder, who has granted bioRxiv a license to display the preprint in perpetuity. It is made available under aCC-BY-NC-ND 4.0 International license.

278 from the STRING database was used (Szklarczyk et al., 2019). The observed high correlations

279 suggest that InterSIMPA is capable to describe biologically relevant promoter-promoter 280 relations by the predictive information hidden within sparse histone mark profiles of an 281 activating mark. Consequently, our approach not only completes the sparse scChIP-seq dataset, 282 but its interpretability-extension is even capable of providing deeper insights into the data.

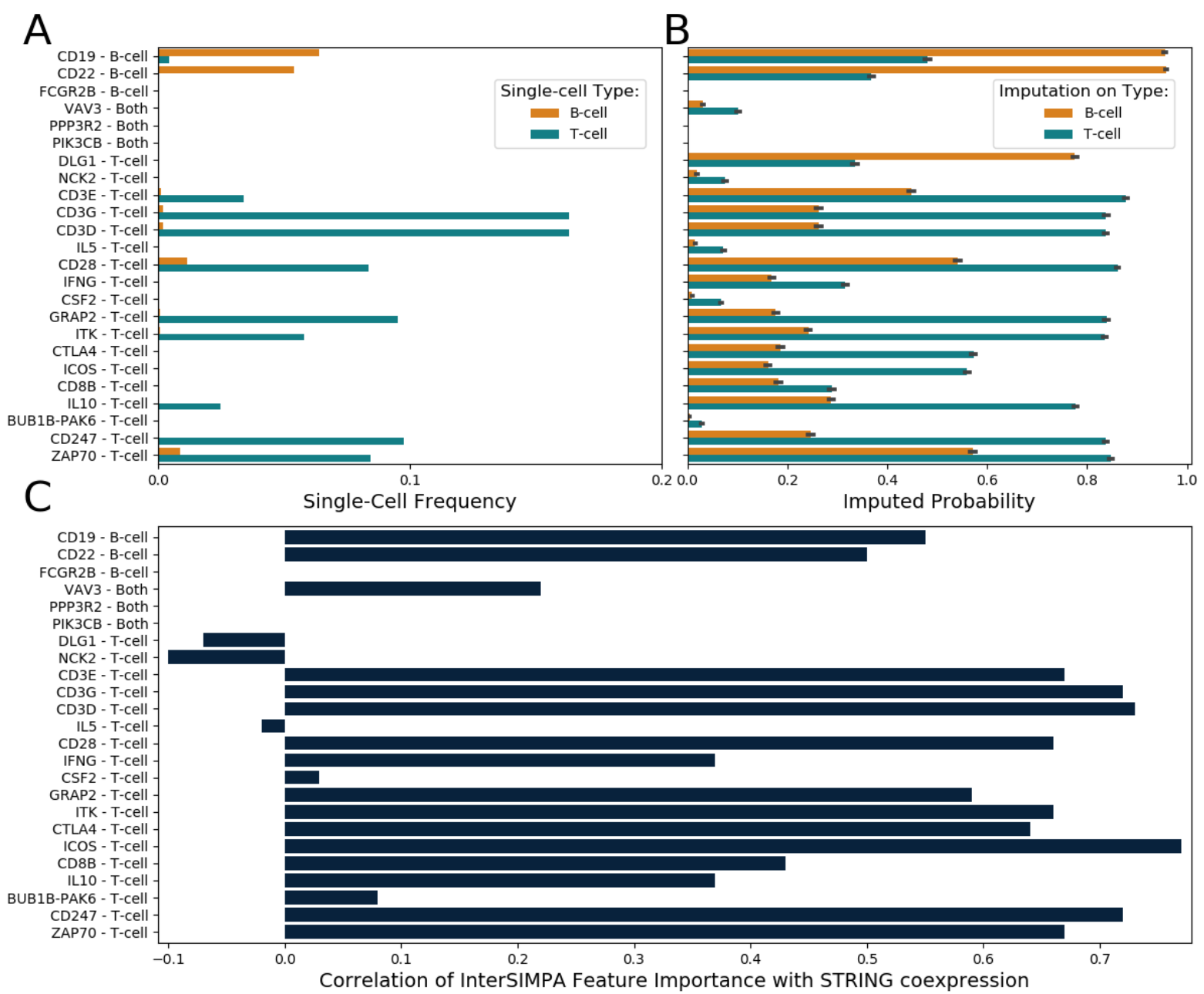

Figure 4 - Pathway related gene analysis using the interpretation of imputation models

286 A. Fraction of single cells for which H3K4me3 binding is observed within the gene's promoter 287 region in the single-cell dataset (orange and blue bars representing B-cells or T-cells, 288 respectively). $y$-axis labels show the gene names and if the gene belongs to the B-cell or T-cell receptor signaling pathway or to both. B. Imputed probability computed by SIMPA for the gene-related promoter regions shown in A. The imputation was applied on numerous single 
cells from the cell-types B-cell (orange) and T-cell (blue). The error bars represent the standard deviation across the imputation runs on different cells. For the majority of genes, the imputed probability is higher within the cell-type that corresponds to the gene's pathway. $\boldsymbol{C}$. Correlation of feature importance and co-expression values. For each model used to impute a promoter (y-axis), the training features (genomic bins) were extracted together with their importance value provided by the Random Forest algorithm and annotated with the nearest gene on the genome. Co-expression values, derived from transcriptomic and proteomic measurements, of those genes with the gene related to the imputed promoter were retrieved from the STRING database. The Pearson correlation coefficient offeature importance and coexpression values is shown (x-axis).

\section{Performance on cell-type clustering and functional analysis} single cell data corresponding to different cell types. For the imputation of a full single-cell dataset, SIMPA was applied for each cell individually. The resulting imputed profiles were corresponding cell-type-specific pathway. Following the investigations of Schreiber et al. (Schreiber, Singh, et al., 2020), we also compared bin probabilities from SIMPA to a simple imputation approach that uses bin frequencies in the reference set (experiments with same protein target) as a probabilistic model without using any machine learning model, called the average interaction method. Additional imputations and randomization tests were applied and compared to better analyze the basic concept of SIMPA (see Supplementary Note 7).

$3145 \mathrm{~kb}$, we present below results on this histone mark and refer to supplementary material for 
in contrast to SIMPA, which takes advantage of information from the reference bulk dataset.

318 After applying a two-dimensional projection on the sparse and imputed datasets, we observed

319 that the separation between the cell types was retained by SIMPA and by the reference-free

320 method, contrary to the average interaction method (Fig. 5A). Moreover, three T-cell outliers

321 were successfully associated to the related cell-type cluster by SIMPA, which achieved a

322

323

324

325

326

327

328

329

330

331

332

333

334

335

336

337

338

339

340 slightly better homogeneity of the clusters in comparison to SCALE (Fig. S6). Dimensionality reduction was done by a combination of principal component analysis (PCA) and t-stochastic neighbor embedding (t-SNE) as suggested by Grosselin et al. on their analysis of sparse data (Grosselin et al., 2019). Different to the suggested procedure, we excluded the cell filtering, as we were interested to observe outliers after imputation.

In order to validate further the algorithmic concept of SIMPA, we implemented two randomization tests in which either the ENCODE reference information was shuffled (Shuffled Reference) or the sparse single-cell input was randomly sampled (Randomized Sparse Input). Additionally, we applied SIMPA on the same data but with different histone marks as target. The selected histone marks were H3K36me3, a histone mark functionally different to H3K4me3, and H3K9ac and H3K27ac, a group of two histone marks functionally related to H3K4me3. These two marks were used together to increase the training data size. From this comparison, we observed that (i) the separation on the projection is lost after removing statistical patterns through shuffling or randomization, (ii) separation quality is moderate with an input mark functionally different from the real mark, and (iii) separation quality stays high using SIMPA with target histone marks functionally similar to the real mark (Fig. 5B). Thus, the most relevant statistical patterns from the reference dataset are identified by both the selection of single-cell-specific regions and the selection of target-specific experiments. Similar observations were made for H3K27me3 although a more compact clustering could be achieved on the SIMPA profiles compared to those from the reference-free method (Fig. S6). 
342 Across several dimensionality reduction procedures applied for the H3K4me3 dataset, SIMPA

343 and the reference-free method were both stable in retaining the cell-type clustering (Fig. S7).

344 From these analyses we additionally conclude that the UMAP method using the Jaccard-Index

345 distance achieves reasonable results when applied directly on the sparse data (in comparison to

346 the common approach in single-cell analysis that uses first a PCA to select dimensions).

347 As pathway enrichment analysis is a common step in ChIP-seq data exploration, we next

348 investigated if enrichment analyses of cell-type-specific pathways for individual single cells

349 improve after applying imputation. We analyzed the sparse profiles and different imputed

350 results with the KEGG pathway analysis function of the Cistrome-GO tool (S. Li et al., 2019).

351 As reported in (Fig. 5C), the original sparse data did not provide enough interaction sites to

352 show a significant pathway enrichment for any of the two cell types. Results from the reference-

353 free strategy showed an improvement but not significant. However, with regions imputed by

354 SIMPA, it was indeed possible to achieve significant enrichment scores and recover the cell-

355 type-specific pathways for most of the cells. These results show that SIMPA is able to integrate

356 functionally relevant information from the reference data in order to impute additional

357 biologically meaningful regions, in contrast to the reference-free method, which is limited to 358 the single-cell dataset. 

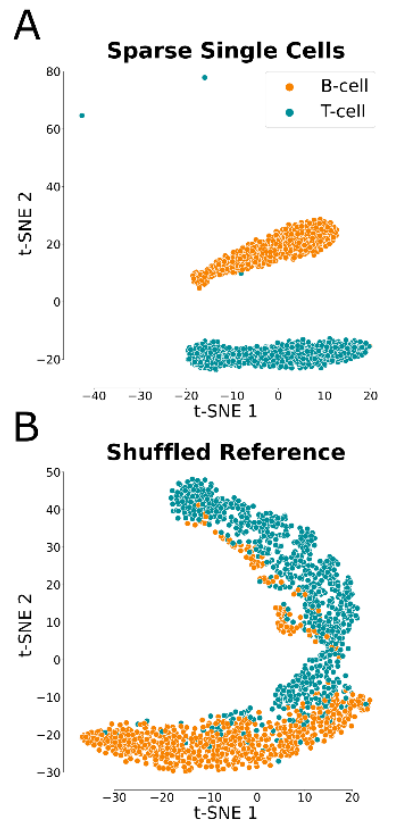

C
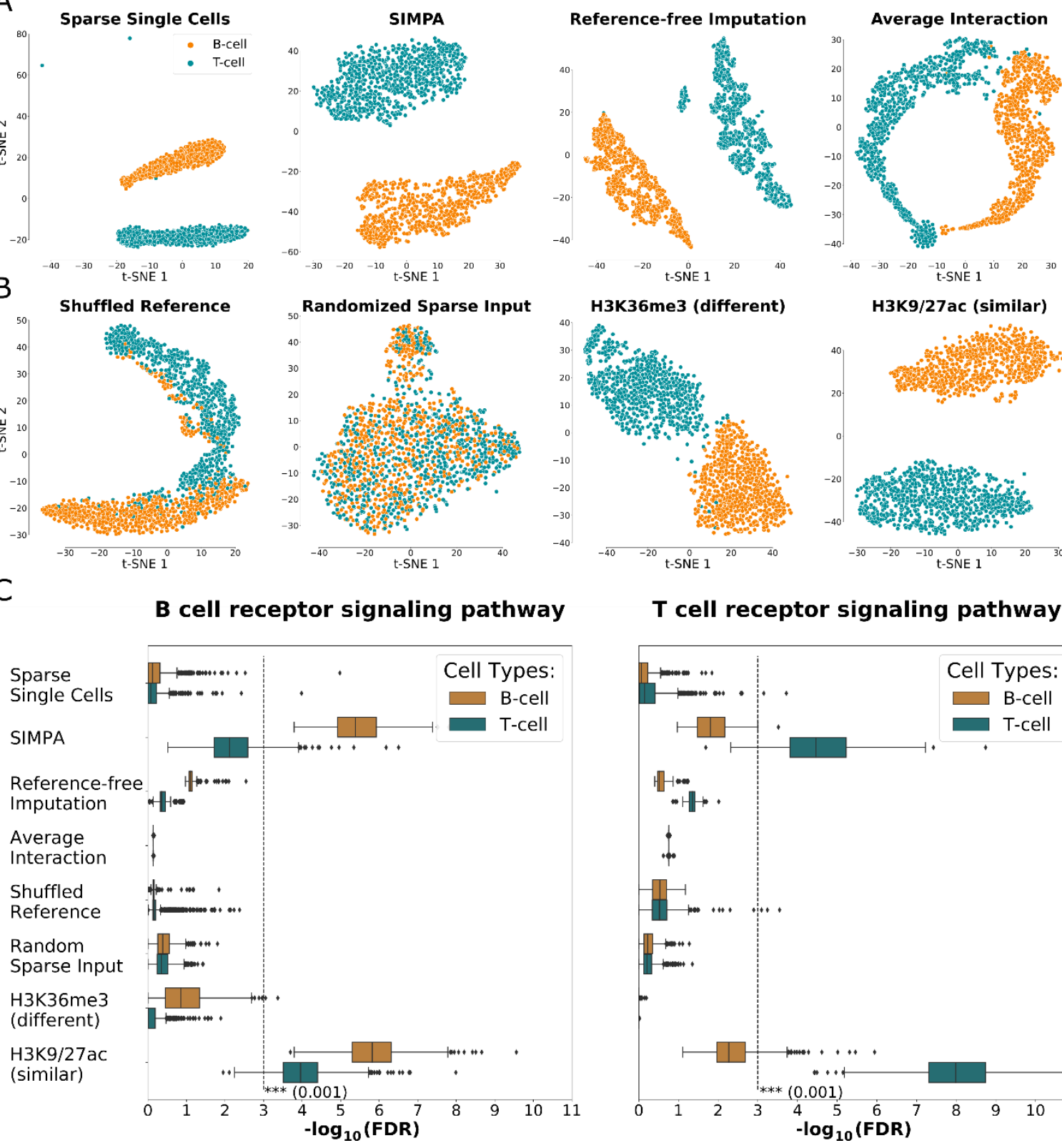

H3K9/27ac (similar)

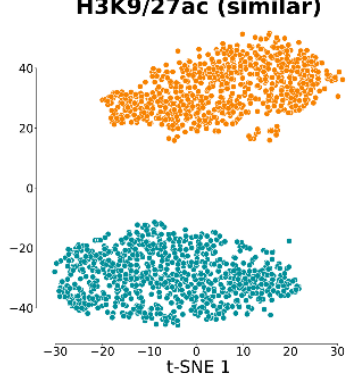

T cell receptor signaling pathway

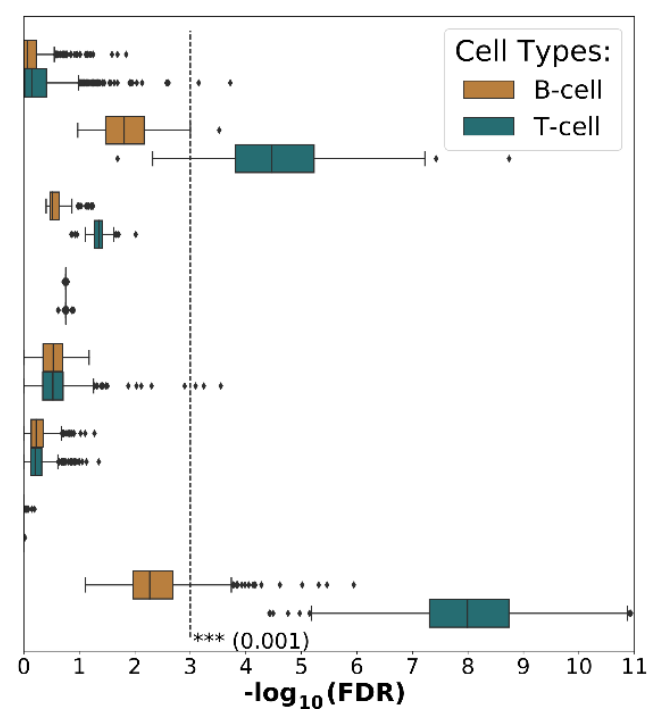

$361 A+B$ Separation of single cells according to cell type. A. Dimensionality reduction analysis applied on the H3K4me3 data derived from ( $i$ ) the sparse single-cell data and three different imputation methods, (ii) SIMPA, (iii) reference-free imputation, and (iv) average interaction

364 based on expected frequencies in the reference set. Results from SIMPA and from the reference-

365 free method achieve the best clustering by separating the single cells (points) by cell types (colors). B. Effects of input modification on SIMPA, (i) using a shuffled reference set or (ii) randomized sparse input data, or using other histone marks as reference instead of H3K4me3, either (iii) the functionally different histone mark H3K36me3, or (iv) the functionally similar 
histone marks H3K9ac and H3K27ac. C. Pathway enrichment analysis. Boxplots show the significance of pathway enrichment analyses of genes annotated by single-cell regions as logtransformed false discovery rate (FDR; $x$-axis). Each dot represents the FDR of one single cell from the results of the different analysis experiments shown in $\boldsymbol{A}+\boldsymbol{B}$ (y-axis). The dashed lines represent the log-transformed significance threshold of an FDR equal to 0.001. Only SIMPA achieves significant results by imputing preferably genomic regions associated with relevant pathway-related genes.

\section{Optimal size of the imputation sets} we imputed a number of bins equivalent to the average number of bins observed across all bulk ChIP-seq profiles from the target-specific reference set. On $5 \mathrm{~kb}$ resolution, the average number of bins of the $\mathrm{H} 3 \mathrm{~K} 4 \mathrm{me} 3$ experiments is 32,584 . However, once the bins are ranked by the imputed probability it is up to the user to alternatively create imputed sets of different sizes. improve the cell-type clustering at the same time enabling the detection of the relevant biological function by a significant enrichment of the correct pathway (for details see

\section{Supplementary Note 8).}

The best cell-type clustering quality, evaluated by the Davies-Bouldin score (Davies and improves the clustering quality compared to the reference-free method. enriched, we observed that in $\sim 50 \%$ of the cells the related pathway is associated when adding $\sim 28,000$ bins (Fig. 6B). After adding more than 32,000 bins, almost all cells have a significant 

analyzing a B-cell and vice versa.
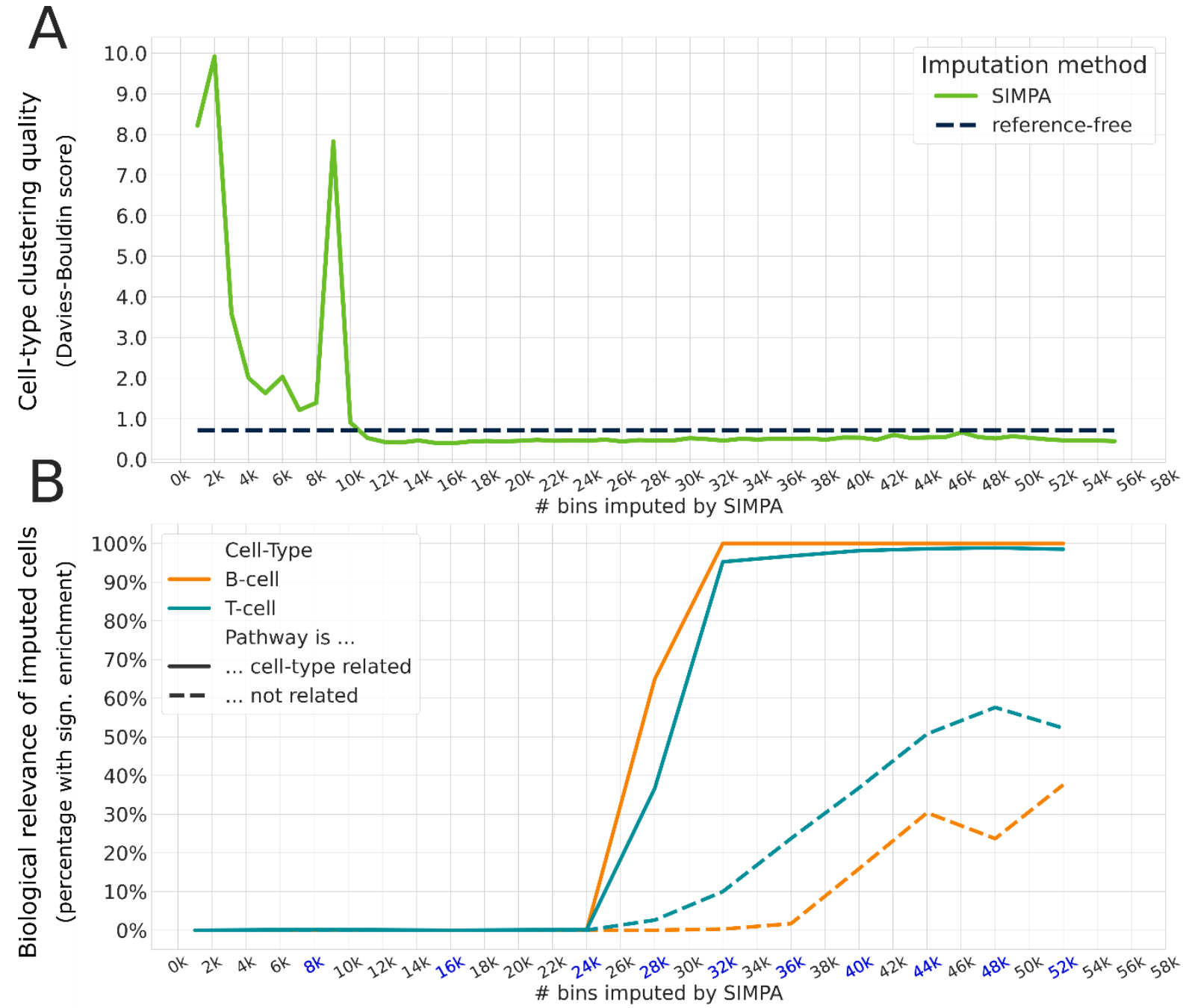

Figure 6 - Clustering quality and pathway enrichment for different sizes

398 A. Clustering quality (y-axis) evaluated with the Davies-Bouldin score (the lower the better) applied on the imputed data after dimensionality reduction as described for Fig. 5 A+B. While the reference-free method derived only one imputed set for all the single cells (dashed black line), we could derive several imputed sets of different sizes using the imputed probabilities from SIMPA (x-axis). B. The pathways under investigation are the B-cell and T-cell receptor signaling pathways. In this way we analyze two pathways surely related or unrelated with the cell-types present in the dataset, B-cell and T-cell. The y-axis describes the percentage of imputed profiles for which a significant enrichment of the aforementioned pathways could be achieved. The dashed lines represent cases for which the unrelated pathway is significantly enriched, which is the T-cell receptor signaling pathway when analyzing a B-cell and vice 
versa. The significance level used is 0.001 similar to the analysis shown in Fig 5C. To reduce the computational resources spent on the pathway enrichment, this was done for ten imputation set sizes (highlighted in blue on the $x$-axis).

\section{Discussion}

After confirming the presence of statistical patterns within the ENCODE bulk ChIP-seq reference data, we show that machine learning models can leverage those patterns for the inference of interaction sites in sparse single-cell ChIP-seq profiles from individual single cells.

Based on the simulations, we could show this also for several cell-type-target combinations even if the experiments related to the cell-type were completely excluded from the training set. In both types of validation (leave-out origin and leave-out cell-type validations), SIMPA was able to capture cell-type-specific patterns even though the reference set was composed of profiles from many different cell-types and tissues, or the cell-type related data was completely excluded. Because the number of available bulk experimental profiles (ENCODE datasets) differs between targets, different training set sizes are available for different targets, with the smallest training set for $\mathrm{H} 3 \mathrm{~K} 9 \mathrm{ac}$ (49 biosamples). Even for training sets of smaller size, the predictive performance remained high, although we expect models to be more reliable the larger the training set. Given that data portals such as ENCODE are still growing, we expect that the model reliability will increase in the future for many targets with a growing number of available reference datasets.

The interpretation of the SIMPA models, done with InterSIMPA applied on a real scChIPseq dataset, allows us to reveal additional information from the ChIP-seq profiles measured within individual cells regarding regions responsible for the imputation. Importantly, leveraging reference data allows us to impute regions that were not present in the single-cell 
dataset at all, in contrast to a reference-free strategy. Considering for example the promoter

434 a high imputed probability from SIMPA. Moreover, for both promoters a high correlation

435 coefficient was achieved within the validation by STRING co-expression values, confirming that our implementation not only answers the question about whether these promoters should

437 be imputed or not, but it additionally reveals valuable information about regulatory relations implied by the single-cell dataset. reference-based imputation strategy compared to the reference-free imputation method. While

441 both algorithms achieve a good separation of the cell types, only with the imputed profiles from

442 SIMPA it was possible to determine the relevant biological function of single cells as shown

443 by the pathway enrichment analysis. This suggests that SIMPA imputes biologically

444 meaningful regions which are of functional relevance and confirms that, even though the

445 training set involves a variety of different tissues and cell-types, SIMPA can find statistical

446 patterns that belong to the correct cell-type. For single-cell datasets which reveal unknown

447 subpopulations of cells, SIMPA could be used to identify active pathways for those cells after

448 imputation. Interestingly, the quality of those results was maintained to some extent when not

449 exactly the same scChIP-seq histone-mark target but functionally related targets were used to

450 define the reference set. This suggests a valuable strategy to be applied for targets with a low

451 availability of public bulk reference profiles.

SIMPA integrates solely datasets from bulk ChIP-seq in order to build the reference set.

453 However, in the future, it will be relevant to integrate other types of data in order to 
455 For example, SCRAT is an analysis tool that summarizes single-cell regulome data using

456 different types of public datasets such as genome annotations or motif databases that could be

457 of interest for the application of SIMPA to transcription factor scChIP-seq profiles (Ji, Zhou

458 and Ji, 2017). The scATAC-seq analysis tool SCATE performs imputation of missing regions

459 integrating different types of public datasets (e.g. co-activated cis-regulatory elements and bulk

460 DNase-seq profiles) (Ji et al., 2020). For future work, such approaches suggest the development

461 of a reference-based method, allowing the imputation for both scChIP-seq and scATAC-seq

462 data, integrating both types of reference data from the corresponding bulk assays and further

463 complementary datasets.

SIMPA's strategy, to train a model for each candidate bin and each single cell, results in its capability to produce highly relevant results and at the same time in its main limitation which

466 is the requirement of a large amount of computational resources. Using a high-performance 467 cluster, the results presented in this manuscript could be obtained within 1-2 days. However, if 468 computational resources are limited, SIMPA offers the opportunity to run the imputation for a 469 selection of cells which, for instance, represent a certain cluster to be analyzed. As shown, cell 470 clusters can be identified even on the sparse profiles using the appropriate method for 471 dimensionality reduction. Importantly, InterSIMPA can also be applied for individual cells, 472 providing interpretable results within seconds of runtime.

\section{Conclusion}

The strategy of SIMPA leveraging bulk ChIP-seq datasets for single-cell sequencing data 475 imputation, is able to complete specifically sparse scChIP-seq data of individual single cells. 476 In comparison to the non-imputed data and a reference-free imputation method, SIMPA was 477 better at recovering cell-type-specific pathways. Furthermore, the interpretability of the 
machine learning models trained for the imputation can be used to reveal biologically important

479 information from a sparse single-cell dataset. Conclusively, we developed an ensemble of

480 computational methods to extract more information from a sparse dataset and impute missing

481 data to better handle data sparsity of scChIP-seq datasets.

\section{References}

Chen, C. et al. (2020) "scRMD: Imputation for single cell RNA-seq data via robust matrix decomposition," Bioinformatics, 36(10), pp. 3156-3161.

Consortium, E. P. and others (2012) "An integrated encyclopedia of DNA elements in the human genome," Nature, 489(7414), pp. 57-74.

Davies, D. L. and Bouldin, D. W. (1979) "A cluster separation measure," IEEE transactions on pattern analysis and machine intelligence, (2), pp. 224-227.

Durham, T. J. et al. (2018) "PREDICTD parallel epigenomics data imputation with cloudbased tensor decomposition," Nature communications, 9(1), p. 1402.

491 Elyanow, R. et al. (2020) "netNMF-sc: leveraging gene-gene interactions for imputation and dimensionality reduction in single-cell expression analysis," Genome research, 30(2), pp. 195-204.

Ernst, J. and Kellis, M. (2015) "Large-scale imputation of epigenomic datasets for systematic annotation of diverse human tissues," Nature biotechnology, 33(4), pp. 364-376.

Grosselin, K. et al. (2019) "High-throughput single-cell ChIP-seq identifies heterogeneity of chromatin states in breast cancer," Nature genetics, 51(6), p. 1060.

Jeong, H. and Liu, Z. (2020) "PRIME: a probabilistic imputation method to reduce dropout effects in single cell RNA sequencing," bioRxiv. 
Li, S. et al. (2019) "Cistrome-GO: a web server for functional enrichment analysis of transcription factor ChIP-seq peaks," Nucleic Acids Research, 47(W1), pp. W206-W211. doi: 10.1093/nar/gkz332.

Peng, T. et al. (2019) "SCRABBLE: single-cell RNA-seq imputation constrained by bulk RNA-seq data," Genome biology, 20(1), p. 88.

Qin, Q. and Feng, J. (2017) "Imputation for transcription factor binding predictions based on deep learning," PLoS computational biology, 13(2), p. e1005403.

Ronen, J. and Akalin, A. (2018) "netSmooth: Network-smoothing based imputation for single cell RNA-seq," F1000Research, 7.

Rotem, A. et al. (2015) "Single-cell ChIP-seq reveals cell subpopulations defined by chromatin state," Nature biotechnology, 33(11), p. 1165.

517 Saito Takaya AND Rehmsmeier, M. (2015) "The Precision-Recall Plot Is More Informative 518 than the ROC Plot When Evaluating Binary Classifiers on Imbalanced Datasets," PLOS ONE, 519 10(3), pp. 1-21. doi: 10.1371/journal.pone.0118432.

520 Schreiber, J., Singh, R., et al. (2020) "A pitfall for machine learning methods aiming to 521 predict across cell types," Genome biology, 21(1), pp. 1-6.

522 Schreiber, J., Durham, T., et al. (2020) "Avocado: a multi-scale deep tensor factorization 523 method learns a latent representation of the human epigenome," Genome biology, 21(1). doi: 10.1186/s13059-020-01977-6.

Schreiber, J., Bilmes, J. and Noble, W. S. (2020) "Completing the ENCODE3 compendium yields accurate imputations across a variety of assays and human biosamples," Genome biology, 21(1), pp. 1-13. single-cell open chromatin profiles," NAR Genomics and Bioinformatics, 2(4), p. Iqaa091. 44(D1), pp. D726-D732. 
Szklarczyk, D. et al. (2019) "STRING v11: protein-protein association networks with increased coverage, supporting functional discovery in genome-wide experimental datasets,"

534 Nucleic acids research, 47(D1), pp. D607-D613.

Tang, W. et al. (2020) "bayNorm: Bayesian gene expression recovery, imputation and

“The ENCODE (ENCyclopedia Of DNA Elements) Project” (2004) Science, 306(5696), pp. 636-640. doi: 10.1126/science.1105136.

Xiong, L. et al. (2019) "SCALE method for single-cell ATAC-seq analysis via latent feature extraction," Nature communications, 10(1), pp. 1-10.

Ye, P. et al. (2020) "scHinter: imputing dropout events for single-cell RNA-seq data with limited sample size," Bioinformatics, 36(3), pp. 789-797.

Yuan, H. et al. (2019) "BindSpace decodes transcription factor binding signals by large-

544 scale sequence embedding," Nature methods, 16(9), pp. 858-861.

545 Zhang, L. and Zhang, S. (2018) "Comparison of computational methods for imputing single546 cell RNA-sequencing data," IEEE/ACM transactions on computational biology and 547 bioinformatics. correlations in two dimensions," Bioinformatics, 36(11), pp. 3588-3589.

553 preprocessed format. Single-cell ChIP-seq data used for the validations was taken from

554 GSE117309. The SIMPA and InterSIMPA software are available at:

555 https://github.com/salbrec/SIMPA. For more information about implementation details and the runtime see Supplementary Note 9. 


\section{$557 \quad$ Competing interests}

558 The authors declare no competing interests.

\section{$559 \quad$ Funding}

560 The project was funded by the Johannes Gutenberg-University Mainz and the international $561 \mathrm{PhD}$ programme of the Institute of Molecular Biology gGmbH, Mainz, Germany.

\section{Authors' contributions}

All authors conceived the study and designed the experiments. SA and TA implemented the

564 algorithm and validation analyses. All authors analyzed the data and interpreted the results. JF and MA co-supervised the research. All authors wrote the article.

\section{Acknowledgements}

We thank Pablo Mier for proofreading the manuscript. We also thank the colleagues that MOGON and advisory services offered by Johannes Gutenberg University Mainz (hpc.unimainz.de), which is a member of the AHRP (Alliance for High-Performance Computing in

572 Rhineland Palatinate, www.ahrp.info) and the Gauss Alliance e.V. The authors gratefully

574 Programme (IPP) of the Institute of Molecular Biology, Mainz, for financial support. We thank

575 Susanne Gerber and Leszek Wojnowski for meaningful discussions. 